\title{
Sensitivity of TLS and minimum norm methods of DOA estimation to errors due to either finite data or sensor gain and phase perturbations
}

\author{
K R SRINIVAS and V U REDDY \\ Department of Electrical Communication Engineering, Indian Institute \\ of Science, Bangalore 560012, India
}

\begin{abstract}
In the DOA (direction of arrival) estimation problem, we encounter either finite data or insufficient knowledge in array characterisation or both. It is therefore important to study how the subspace-based methods perform under these conditions.

In this paper, we first consider the finite data case and establish two results: (i) the total least-squares approach to the linear prediction method (which we refer to as TLS-FLP method) is equivalent to the minimum norm (min. norm), method and (ii) the TLS-FBLP method yields a $3 \mathrm{~dB}$ lower mean-square error (MSE) in the DOA estimates as compared to the TLS-FLP method.

Next, we consider the asymptotic performance of the min. norm method in the presence of sensor gain and phase perturbations, and derive the expressions for the MSE in the DOA estimates assuming an uniform linear array. For the special case of a single source, we also obtain a simple and explicit expression for the MSE which, when compared with the corresponding result for the MUSIC algorithm, shows that the min. norm method is more sensitive than the MUSIC when the number of sensors exceeds 2 .

Computer simulations are included to support the theoretical predictions.
\end{abstract}

Keywords. Sensitivity; minimum norm method; emitter location; sensor errors.

\section{Introduction}

Eigenstructure-based techniques for direction of arrival (DOA) estimation of narrowband emitters, pioneered by Pisarenko (1973) and generalised by Schmidt (1986) and Bienvenu \& Kopp (1983), have recently been receiving a lot of attention. The method of Schmidt, often called MUSIC (Multiple Signal Classification), has been the subject of active research in recent years. The other methods such as the minimum-norm (min. norm) method (Kumaresan \& Tufts 1983) and ESPRIT (Paulraj et al 1985) fall in this class of eigenstructure-based (or subspace-based) methods. While MUSIC is applicable to arrays with arbitrary geometry and arbitrary sensor directional characteristics, a variant of this called root-MUSIC (Barabell 1983, pp. 336-339), is 
applicable to uniform linear arrays, while ESPRIT is applicable to certain special arrays. The distinct feature of all the subspace-based methods is that they yield high resolution and asymptotically exact results for the DOA. Another feature of these methods is that they require exact characterisation of the array in terms of its geometry and sensor gain and phase. In practice, however, we only have finite data to work with and also the characterisation of the array may be different from the nominal (i.e. assumed) value either because of erroneous characterisation of its geometry or errors in characterising the sensor gain and phase or both.

In the context of spectral estimation with finite data, several linear predictionbased formulations such as forward linear prediction (FLP), forward-backward linear prediction (FBLP) and modified FBLP (MFBLP) (Tufts \& Kumaresan 1982) have been proposed. Recently, a variant of the MFBLP (Reddy \& Srinivas 1989) and total least squares (TLS) (Rahman \& Yu 1987) method, which are essentially identical but motivated differently, have been suggested for frequency estimation. These methods can be straightaway extended to the DOA estimation of narrowband emitters in finite data. Application of FBLP and MFBLP to the DOA estimation problem is discussed in Kumaresan \& Tufts (1983) while that of TLS is discussed in Srinath (1990). Srinath (1990) compares finite data performance of the TLS method with that of root-MUsIC and min. norm method using simulations.

Kaveh \& Barabell (1986) were probably the first to study the performance of MUSIC and min. norm methods in finite data. Recently, Rao \& Hari (1989) analysed the finite data performance of the root-MUSIC and min. norm methods and derived expressions for the mean-square error (MSE) in the DOA estimates. In their analysis (Kaveh \& Barabell 1986; Rao \& Hari 1989a, b), they assumed exact characterisation of the array. More recently, Srinath \& Reddy (1991) analysed the performance of MUSIC with sensor gain and phase perturbations and derived expressions for MSE in DOA estimates. Their analysis is asymptotic in that no finite data errors are considered.

In this paper, we first consider the finite data case and derive two results. The first result shows that the min. norm method and the TLS approach to the FLP method (which we refer to as TLS-FLP method) yield coefficient vectors that are complex conjugates of each other implying that the DOA estimates are same in magnitude but opposite in sign. The second result shows that the MSE in the DOA estimates obtained by the TLS-FBLP method is smaller than that of TLS-FLP by $3 \mathrm{~dB}$. Next, we consider the asymptotic performance of the min. norm method in the presence of sensor gain and phase perturbations and derive expressions for MSE in DOA estimates assuming an uniform linear array. These expressions reduce to a simple and explicit form in the one-source case. Computer simulations are used to verify the theoretical predictions.

Section 2 introduces briefly the background material and the notation. In $\S 3$, we show that TLS-FLP and min. norm methods yield coefficient vectors which are complex conjugates of each other and TLS-FBLP yields lower MSE as compared to that of TLS-FLP. The expressions for the MSE in the DOA estimates with min. norm method in the presence of sensor gain and phase perturbations are derived in $\$ 4$. Section 5 gives simulation results and finally, $\$ 6$ concludes the paper.

\section{Background and notation}

Assume that plane waves emitted by $D$ narrowband sources impinge on a linear array consisting of $M$ equispaced omnidirectional sensors, and the DOA of these sources 
relative to the normal to the array are $\left\{\theta_{1}, \theta_{2}, \ldots, \theta_{D}\right\}$. The output of the $m$ th sensor can be expressed as

$$
r_{m}(t)=\sum_{k=1}^{D} s_{k}(t) \exp \left[-j \omega_{0}(m-1) \tau_{k}\right]+n_{m}(t)
$$

where $s_{k}(\cdot)$ denotes the signal emitted by the $k$ th sensor as observed at sensor one, $\omega_{0}$ is the centre radian frequency of the sources, and $n_{m}(\cdot)$ is the additive noise which is assumed to be a zero-mean, stationary and white complex Gaussian random process that is uncorrelated from sensor to sensor. The signals are assumed to be zero-mean, stationary and complex Gaussian random processes that are arbitrarily correlated (but not fully correlated). The interelement path delay is given by $\tau_{k}=(\Delta / c) \sin \left(\theta_{k}\right)$ where $\Delta$ is the sensor spacing and $c$ is the propagation velocity of the wave.

Let $\mathbf{r}^{T}(t)=\left[r_{1}(t), r_{2}(t), \ldots, r_{m}(t)\right]$ be the simultaneously sampled vector of array signal, and similarly define $\mathbf{s}(\cdot)$ and $\mathbf{n}(\cdot)$. The array signal vector can then be expressed as

$$
\mathbf{r}(t)=\mathbf{A s}(t)+\mathbf{n}(t)
$$

where $\mathbf{A}=\left[\mathbf{a}\left(\theta_{1}\right) \cdots \mathbf{a}\left(\theta_{D}\right)\right]$ with $\mathbf{a}\left(\theta_{k}\right), k=1,2, \ldots, D$, defined as (with $\lambda$ denoting the wavelength)

$$
\mathbf{a}\left(\theta_{k}\right)=\left[1, \exp \left[-j(2 \pi \Delta / \lambda) \sin \left(\theta_{k}\right)\right], \ldots, \exp \left[-j(M-1)(2 \pi \Delta / \lambda) \sin \left(\theta_{k}\right)\right]\right]^{T} .
$$

The covariance matrix of the array signal vector $\mathbf{r}(t)$ is given by

$$
\mathbf{R}=E\left[\mathbf{r}(t) \mathbf{r}^{+}(t)\right]=\mathbf{A S A}^{+}+\sigma^{2} \mathbf{I}
$$

where the superscript ' + ' denotes conjugate transpose, $\mathbf{S}=E\left[\mathbf{s}(t) \mathbf{s}^{+}(t)\right]$ and $\sigma^{2}$ is the variance of the additive noise. Let $\lambda_{1} \geqslant \lambda_{2} \geqslant \cdots \geqslant \lambda_{D}>\lambda_{D+1}=\cdots=\lambda_{M}=\sigma^{2}$ denote the eigenvalues of $\mathbf{R}$ and $\mathbf{e}_{1}, \mathbf{e}_{2}, \ldots, \mathbf{e}_{M}$ denote the corresponding eigenvectors. If the matrices $\mathbf{E}_{s}$ and $\mathbf{E}_{n}$ are formed as

and

$$
\mathrm{E}_{\mathrm{s}}=\left[\mathrm{e}_{1}, \mathrm{e}_{2}, \ldots, \mathrm{e}_{D}\right]
$$

$$
\mathbf{E}_{n}=\left[\mathbf{e}_{D+1}, \mathbf{e}_{D+2}, \ldots, \mathbf{e}_{M}\right]
$$

then the linear span of $\mathbf{E}_{\boldsymbol{s}}$, known as the signal subspace, is the same as that spanned by the columns of $\mathbf{A}$. The linear span of $\mathbf{E}_{n}$, known as the noise subspace, is the orthogonal complement of the signal subspace. Then

$$
\mathbf{E}_{n}^{+} \mathbf{a}\left(\theta_{k}\right)=0, \quad k=1,2, \ldots, D
$$

\subsection{The minimum norm method}

In this method (Kumaresan \& Tufts 1983), a coefficient vector $\mathbf{d}=\left[d_{1}, d_{2} \cdots d_{M}\right]^{T}$ is chosen as a linear combination of the noise eigenvectors with $d_{1}=1$. It then satisfies

$$
\mathbf{E}_{s}^{+} \mathbf{d}=0
$$


and hence

$$
\mathrm{a}^{+}\left(\theta_{k}\right) \mathrm{d}=0, \quad k=1,2, \ldots, D
$$

Equation (8) implies that the $D$ roots of the polynomial

$$
D(z)=\sum_{k=1}^{M} d_{k} z^{k-1}
$$

will correspond to $z_{k}=\exp \left[j(2 \pi \Delta / \lambda) \sin \left(\theta_{k}\right)\right], k=1,2, \ldots, D$.

Express

$$
\mathbf{E}_{s}=\left[\begin{array}{l}
\mathbf{g}^{T} \\
\tilde{\mathbf{E}}_{s}
\end{array}\right],
$$

where $\mathbf{g}^{T}$ is the first row of $\mathbf{E}_{s}$ and $\tilde{\mathbf{E}}_{\mathrm{s}}$ is the rest of the matrix, and

$$
\mathbf{d}=\left[\begin{array}{l}
1 \\
\tilde{d}
\end{array}\right] \text {. }
$$

Then (7) can be rewritten as

$$
\mathbf{g}^{*}=-\tilde{\mathbf{E}}_{s}^{+} \tilde{\mathbf{d}}
$$

If $M=D+1$, we will have a unique solution for $\mathrm{d}$ and the polynomial (9) will be of order $D$ yielding $D$ signal zeros. However, if $M>D+1$, there will be several solutions for $\mathbb{d}$ and each solution vector, when used in (9), gives $D$ signal zeros and $M-1-D$ extraneous zeros. Kumaresan \& Tufts (1983) argued that if a mimimum-norm solution for $\tilde{d}$ is used, then the extraneous zeros will be forced to lie inside the unit circle thereby minimizing the effects of noise. The solution vector so selected is given by

$$
d=\left[\begin{array}{c}
1 \\
-\tilde{\mathbf{E}}_{\mathbf{s}} \mathbf{g}^{*} /\left(1-\mathbf{g}^{T} \mathbf{g}^{*}\right)
\end{array}\right]
$$

We form the polynomial $D(z)$ using this $\mathrm{d}$ and choose the $D$ zeros that are closest to the unit circle and estimate the DOA from the angles of these zeros.

In finite data case, we work with the estimate of $\mathbf{R}, \hat{\mathbf{R}}=(1 / N) \Sigma_{i=1}^{N} \mathbf{r}\left(t_{i}\right) \mathbf{r}^{+}\left(t_{i}\right)$, where $N$ is the number of snapshots, and consequently, the signal and noise subspaces we derive from $\hat{\mathbf{R}}$ become the estimates of the true ones.

\section{Finite-data performance analysis of TLS and minimum norm methods}

In this section, we first describe briefly the TLS approach and then establish equivalence between the minimum norm and TLS-FLP methods. Next, we show how TLS-FBLP yields $3 \mathrm{~dB}$ lower MSE in DOA estimates compared to that of TLS-FLP.

\subsection{TLS-FLP and its equivalence to the minimum norm method}

Let $r_{i}(n)$ denote the $i$ th sensor output at the $n$th snapshot time. The observed data vector is then expressed as

$$
\mathbf{r}_{n}=\left[r_{1}(n), r_{2}(n), \ldots, r_{M}(n)\right]^{T}
$$


Using these data vectors, we form a set of forward linear prediction equations as

$$
\left[\begin{array}{ccc}
r_{2}(1) & \cdots & r_{M}(1) \\
r_{2}(2) & \cdots & r_{M}(2) \\
\vdots & \cdots & \vdots \\
r_{2}(N) & \cdots & r_{M}(N)
\end{array}\right]\left[\begin{array}{c}
h_{2} \\
h_{3} \\
\vdots \\
\underbrace{h_{M}}_{\tilde{h}}
\end{array}\right] \approx-\left[\begin{array}{c}
r_{1}(1) \\
r_{1}(2) \\
\vdots \\
\underbrace{r_{1}(N)}_{b}
\end{array}\right]
$$

which can be expressed in compact form as

$$
\mathbf{Q} \tilde{\mathbf{h}} \approx-\mathbf{b} \text {. }
$$

The approximate sign arises because of the presence of noise in the data. The TLS solution of $\tilde{\mathbf{h}}$ is as follows.

Consider perturbations of both $\mathbf{b}$ and $\mathbf{Q}$ such that

$$
(\mathbf{Q}+F) \tilde{\mathbf{h}}=-(\mathbf{b}+r)
$$

which can be arranged in the following form:

or

$$
([\mathbf{b} \mid \mathbf{Q}]+[r \mid F])\left[\begin{array}{c}
1 \\
\tilde{\mathbf{h}}
\end{array}\right]=0
$$

$$
(\mathbf{B}+H) \mathbf{h}=0 \text {, }
$$

where

$$
\mathbf{B}=[\mathbf{b} \mid \mathbf{Q}], \quad H=[r \mid F], \quad \mathbf{h}=\left[\begin{array}{c}
1 \\
\tilde{h}
\end{array}\right] .
$$

The TLS solution (Golub \& Van Loan 1980) to (18) is the solution vector h such that $\|H\|_{F}$ is minimum and $(b+r)$ lies in the range of $(Q+F)$, where $\|\cdot\|_{F}$ denotes the Frobenius norm. Now, replacing $(B+H)$ by $B_{1}$, the lower $D$-rank approximate of $B$, is equivalent to choosing $H$ as $\left(\mathbf{B}-\mathbf{B}_{1}\right)$ which has the minimum F-norm according to the definition of $B_{1}$. Thus, the TLs solution of $(18)$ is equivalent to seeking a solution to

$$
\mathbf{B}_{\mathbf{1}} \mathbf{h}=\mathbf{0}
$$

We may point out here that this is in the same form as the one arrived at in the variant of the modified FBLP case (Reddy \& Srinivas 1989), though the motivation there was to minimize the effect of noise on the data matrix $(Q)$ and the predicted data vector (b).

When $M>D+1,(20)$ gives several solutions for $\mathbf{h}$ and, as in the minimum norm method, we choose the minimum-norm solution (which is the so-called TLS solution). For the purpose of establishing the equivalence between the TLS-FLP and the minimum norm methods, let us express this solution in terms of the singular vectors of $\mathbf{B}$.

From the singular value decomposition (SVD) of $B$, we can express the lower $D$-rank approximate $B_{1}$ as

$$
\mathbf{B}_{1}=\mathbf{U}_{s} \boldsymbol{\Sigma}_{s} \mathbf{V}_{s}^{+}
$$

where $U_{s}=\left[u_{1} u_{2} \cdots u_{D}\right]$ and $V_{s}=\left[v_{1} v_{2} \cdots v_{D}\right]$ are the matrices containing the left and 
right signal singular vectors of $\mathbf{B}$, respectively, and $\Sigma_{\mathrm{a}}$ is the $D \times D$ diagonal matrix with the signal singular values as diagonal elements. Thus, (20) can be expressed as

$$
\mathbf{U}_{s} \Sigma_{s} \mathbf{V}_{s}^{+} \mathbf{h}=0
$$

Since $\mathbf{U}_{s}$ is of full column rank and $\boldsymbol{\Sigma}_{s}$ is a full-rank diagonal matrix, (22) implies that

$$
\mathbf{V}_{s}^{+} \mathbf{h}=0
$$

which is in the same form as (7). The minimum norm solution for $h$ is then given by

where

$$
\mathbf{h}=\left[\begin{array}{c}
1 \\
-\tilde{\mathbf{V}}_{\mathbf{s}} \mathbf{f}^{*} /\left(1-\mathbf{f}^{T} \mathbf{f}^{*}\right)
\end{array}\right]
$$

$$
\mathbf{V}_{s}=\left[\begin{array}{c}
\mathbf{f}^{T} \\
\tilde{\mathbf{V}}_{s}
\end{array}\right]
$$

with $\mathbf{f}^{T}$ as the first row of $\mathbf{V}_{s}$ and $\tilde{\mathbf{V}}_{s}$ as the rest of the matrix.

Now recall that the right singular vector matrix $\mathbf{V}$ is the eigenvector matrix of $(1 / N) B^{+}$B. But

$$
(1 / N) \mathbf{B}^{+} \mathbf{B}=(1 / N)[\mathbf{b} \mid \mathbf{Q}]^{+}[\mathbf{b} \mid \mathbf{Q}]=(1 / N) \sum_{i=1}^{N} \mathbf{r}^{*}(i) \mathbf{r}^{T}(i)=\hat{\mathbf{R}}^{*} .
$$

Hence, in the finite data case, $\mathbf{V}_{s}$, the signal part of $\mathbf{V}$, is the same as $\hat{\mathbf{E}}_{s}^{*}$, the signal part of $\hat{E}^{*}$. Consequently, the coefficient vector given by (24) is the complex conjugate of the vector d given by (13). Thus, the signal zeros of the polynomial $D(z)$ (cf. (9)) with $\left\{h_{i}\right\}$ as the coefficients are the complex conjugates of those obtained with $\left\{d_{i}\right\}$. Hence the DOA estimates obtained by the TLS-FLP and the minimum norm methods are the same in magnitude but opposite in sign. It therefore follows that the MSE in the DOA estimates (in the finite data case) is the same in both cases.

\section{$3.2 T$ TLS-FBLP and its finite data performance}

The prediction equations in the case of FBLP are arranged both in the forward and reverse directions as

$$
\left[\begin{array}{cccc}
r_{1}(1) & r_{2}(1) & \cdots & r_{M}(1) \\
r_{1}(2) & r_{2}(2) & \cdots & r_{M}(2) \\
\vdots & \vdots & \cdots & \vdots \\
r_{1}(N) & r_{2}(N) & \cdots & r_{M}(N) \\
r_{M}^{*}(1) & r_{M-1}^{*}(1) & \cdots & r_{1}^{*}(1) \\
r_{M}^{*}(2) & r_{M-1}^{*}(2) & \cdots & r_{1}^{*}(2) \\
\vdots & \vdots & \cdots & \vdots \\
r_{M}^{*}(N) & r_{M-1}^{*}(N) & \cdots & r_{1}^{*}(N)
\end{array}\right]\left[\begin{array}{c}
1 \\
w_{2} \\
w_{3} \\
\vdots \\
w_{M}
\end{array}\right] \approx\left[\begin{array}{c}
0 \\
0 \\
0 \\
\vdots \\
0
\end{array}\right] .
$$

Following the steps as in the case of TLS-FLP, we can show that the coefficient vector is given by

$$
\mathbf{w}=\left[\begin{array}{c}
1 \\
-\tilde{\mathbf{C}}_{s} \mathbf{q}^{*} /\left(1-\mathbf{q}^{T} \mathbf{q}^{*}\right)
\end{array}\right]
$$


where

$$
\mathrm{C}_{s}=\left[\begin{array}{c}
\mathbf{q}^{T} \\
\widetilde{\mathbf{C}}_{s}
\end{array}\right],
$$

with $\mathbf{q}^{\boldsymbol{T}}$ as the first row of $\mathbf{C}_{s}$ and $\widetilde{\mathbf{C}}_{s}$ as the rest of the matrix. Here, $\mathbf{C}_{s}$ is the right signal singular vector matrix of the FBLP data matrix. But, this is same as the signal eigenvector matrix of $\hat{\mathbf{R}}_{\text {FBLP. }}$.

Now, the data covariance matrix $\hat{\mathbf{R}}_{\mathrm{FBLP}}$ is given by

$$
\hat{\mathbf{R}}_{\mathrm{FBLP}}=(1 / 2 N) \mathbf{B}_{\mathrm{FBLP}}^{+} \mathbf{B}_{\mathrm{FBLP}} .
$$

In the limit, as $N \rightarrow \infty$, we obtain

$$
\mathbf{R}_{\mathrm{FBLP}}=\frac{1}{2}\left(\mathbf{R}^{*}+\tilde{\mathbf{I}} \mathbf{\mathbf { I }}\right),
$$

where $\mathbf{R}$ is the true covariance matrix and $\tilde{\mathbf{I}}$ is the exchange matrix. For the case of a uniform linear array, $\tilde{\mathbf{I}} \tilde{\mathbf{I}}$ becomes $\mathbf{R}^{*}$ and hence

$$
\mathbf{R}_{\text {FBLP }}=\mathbf{R}^{*} \text {. }
$$

In the finite data performance analysis, we decompose $\hat{\mathbf{R}}_{\mathrm{FBLP}}$ as

$$
\hat{\mathbf{R}}_{\mathrm{FBLP}}=\mathbf{R}^{*}+\varepsilon \mathbf{\Psi},
$$

and use the identities of the type given below (see Kaveh \& Barabell 1986 for details)

$$
\overline{\alpha^{+} \varepsilon \overline{\mathbf{T} \beta \gamma^{+} \varepsilon \boldsymbol{\Psi} \delta}}=\alpha^{+} \mathbf{R}^{*} \delta \gamma^{+} \mathbf{R}^{*} \beta / 2 N,
$$

where $\alpha, \beta, \gamma, \delta$ are vectors of suitable dimension. This identity, when used in deriving the expressions for MSE, gives a factor $2 N$ in the denominator as against $N$ in the case of TLS-FLP, thus resulting in $3 \mathrm{~dB}$ reduction in the MSE compared to that in the TLS-FLP method. The simulation results given in $\$ 5$ corroborate this.

\section{Analysis of the minimum norm method with sensor gain and phase perturbations}

In this section, we derive expressions for the MSE in the DOA estimates which the minimum norm method yields in the presence of sensor gain and phase perturbations. The analysis is asymptotic, i.e., infinite data is assumed.

The sensor gain and phase errors are modelled as zero-mean random variables that are independent from sensor to sensor. We also assume, without any loss of generality, that the nominal gain of each sensor is unity. Thus, the complex gain $g_{i}$ of the $i$ th sensor can be expressed as

$$
g_{i}=\left(1+\Delta a_{i}\right) \exp \left(j \Delta p_{i}\right) \approx 1+\Delta a_{i}+j \Delta p_{i} \triangleq 1+\Delta g_{i}
$$

where the amplitude (or gain) error $\Delta a_{i}$ and the phase error $\Delta p_{i}$ are assumed to be small.

The perturbed covariance matrix (the asymptotic data covariance matrix with the actual array) is then given by

$$
\check{\mathbf{R}}=\mathbf{G A S A}^{+} \mathbf{G}^{+}+\sigma^{2} \mathbf{I},
$$


where $\mathbf{G}=\mathbf{I}+\Delta \mathbf{G}$ with $\Delta \mathbf{G}$ denoting the diagonal matrix whose elements are $\Delta g_{1}$, $\Delta g_{2}, \ldots, \Delta g_{M}$. We now decompose $\check{\mathbf{R}}$ into the true (i.e., nominal) covariance matrix and a perturbation matrix $\Delta \mathbf{R}$

where

$$
\check{\mathbf{R}}=\mathbf{R}+\Delta \mathbf{R},
$$

$$
\Delta \mathbf{R}=\Delta \mathbf{G A S A}^{+}+\mathbf{A S A}^{+} \Delta \mathbf{G}^{+}+\Delta \mathbf{G A S A}{ }^{+} \Delta \mathbf{G}^{+} .
$$

The perturbed signal eigenvectors (i.e., signal eigenvectors of $\check{\mathbf{R}}$ ) can be written as

$$
\check{\mathbf{e}}_{i}=\mathbf{e}_{i}+\eta_{i}, \quad i=1,2, \ldots, D,
$$

where $\eta_{i}$ is the first order perturbation of the $i$ th eigenvector (see Srinath \& Reddy 1991)

$$
\eta_{i}=-\frac{1}{2} \sum_{l=1, l \neq i}^{M} \frac{\mathbf{e}_{l}^{+} \Delta \mathbf{R e}_{i} \mathbf{e}_{i}^{+} \Delta \mathbf{R e}_{l}}{\left(\lambda_{i}-\lambda_{l}\right)^{2}} \mathbf{e}_{i}+\sum_{l=1, l \neq i}^{M} \frac{\mathbf{e}_{l}^{+} \Delta \mathbf{R e}_{i}}{\left(\lambda_{i}-\lambda_{l}\right)} \mathbf{e}_{l} .
$$

If we now express the perturbed signal subspace as

then

$$
\check{\mathbf{E}}_{s}=\mathbf{E}_{s}+\Delta \mathbf{E}_{s} \text {, }
$$

$$
\Delta \mathbf{E}_{s}=\left[\eta_{1}, \eta_{2}, \ldots, \eta_{D}\right] .
$$

To derive the expression for the MSE in the DOA estimates, we begin with the following expression for $\overline{\left(\Delta \omega_{i}\right)^{2}}$ (see Rao \& Hari 1989a):

$$
\overline{\left(\Delta \omega_{i}\right)^{2}}=\frac{1}{2}\left[\overline{\left(\left.\Delta z_{i}\right|^{2}\right.}-\operatorname{Re}\left\{\left(z_{i}^{*}\right)^{2} \overline{\left(\Delta z_{i}\right)^{2}}\right\}\right],
$$

where $\Delta z_{i}$ is given by (the overbar denotes expectation)

$$
\Delta z_{i}=\left[-\sqrt{M} / \prod_{l=1, l \neq i}^{M-1}\left(1-z_{l}^{-1} z_{i}\right)\right] \mathbf{V}^{\prime+}\left(\omega_{i}\right) \Delta \tilde{\mathrm{d}} .
$$

Here, $\mathbf{V}^{\prime} \mathbf{T}\left(\omega_{i}\right)=(1 / \sqrt{M})\left[0,1, e^{-j \omega_{i}}, \ldots, e^{-j(M-2) \omega_{i}}\right]$ with $\omega_{i}=(2 \pi \Delta / \lambda) \sin \left(\theta_{i}\right), z_{i}$ is the $i$ th root of the polynomial $D(z)(\mathrm{cf}(9))$ and $\Delta \bar{d}$ is the perturbation in the reduced-dimension coefficient vector $\mathbb{d}$, where $\mathbb{d}$ is as given in (11). Noting that the first element of $\Delta d$ is zero, $\Delta z_{i}$ can be rewritten as

$$
\Delta z_{i}=\left[-\sqrt{M} e^{-j \omega_{i}} / \prod_{l=1, l \neq i}^{M-1}\left(1-z_{l}^{-1} z_{i}\right)\right] \mathbf{V}^{+}\left(\omega_{i}\right) \Delta \mathrm{d},
$$

where $\mathbf{V}\left(\omega_{i}\right)=(1 / \sqrt{M})\left[1, e^{-j \omega_{i}}, \ldots, e^{-J(M-1) \omega_{i}}\right]^{T}$. Hereafter, we denote $\mathbf{V}^{\prime}\left(\omega_{i}\right)$ and $\mathbf{V}\left(\omega_{i}\right)$ by $\mathbf{V}^{\prime}$ and $\mathbf{V}$, respectively, for convenience.

From (42), we have

$$
\overline{\left|\Delta z_{i}\right|^{2}}=\left[M\left|\prod_{l=1, l \neq i}^{M-1}\right| 1-\left.z_{l}^{-1} z_{l}\right|^{2}\right] \mathbf{V}^{+} \overline{\Delta \mathrm{d} \Delta \mathbf{d}^{+}} \mathbf{V}
$$


Substituting the result for $\Delta \mathrm{d}$ from (A4) (see appendix A) ${ }^{1}$, we obtain

$$
\overline{\left|\Delta z_{i}\right|^{2}}=\left[M / \prod_{l=1, l \neq i}^{M-1}\left|1-z_{l}^{-1} z_{i}\right|^{2}\right] \mathbf{V}^{+} \mathbf{E}_{s} \overline{\Delta \mathbf{E}_{s}^{+} \mathrm{dd}^{+} \Delta \mathbf{E}_{s}} \mathbf{E}_{s}^{+} \mathbf{V}
$$

We now evaluate $\overline{\Delta \mathbf{E}_{s}^{+}} \overline{\mathrm{dd}^{+}} \overline{\Delta \mathbf{E}_{s}}$, which we denote by $\mathbf{P}_{1}$ for convenience. $\mathbf{P}_{1}$ is a $D \times D$ matrix whose $(i, j)$ th element is given by

$$
P_{1}(i, j)=\overline{\eta_{i}^{+} \mathrm{dd}^{+} \eta_{j}}=\mathrm{d}^{+} \overline{\eta_{j} \eta_{i}^{+}} \mathrm{d} .
$$

Substituting for $\eta_{i}$ from (38) in (45) and neglecting the terms containing three or more $\Delta \mathbf{R}$ 's, we get

$$
P_{1}(i, j)=\sum_{\substack{m=1 \\ m \neq j}}^{M} \sum_{\substack{n=1 \\ n \neq i}}^{M} \frac{\mathbf{e}_{m}^{+} \overline{\Delta \mathbf{R e}_{j} \mathbf{e}_{i}^{+} \Delta \mathbf{R}} \mathbf{e}_{n}}{\left(\lambda_{j}-\lambda_{m}\right)\left(\lambda_{i}-\lambda_{n}\right)} \mathbf{d}^{+} \mathbf{e}_{m} \mathbf{e}_{n}^{+} \mathbf{d}
$$

Since $\mathbf{d}$ is orthogonal to the signal subspace, the above equation simplifies to

$$
P_{1}(i, j)=\sum_{m=\bar{D}+1}^{M} \sum_{n=\bar{D}+1}^{M} \frac{\mathbf{e}_{m}^{+} \overline{\Delta \operatorname{Re}_{j} \mathbf{e}_{i}^{+} \Delta R} \mathbf{e}_{n}}{\left(\lambda_{i}-\sigma^{2}\right)\left(\lambda_{j}-\sigma^{2}\right)} \mathbf{d}^{+} \mathbf{e}_{m} \mathbf{e}_{n}^{+} \mathbf{d}
$$

Substituting (36) in (47) and simplifying, we get

$$
P_{1}(i, j)=\sum_{m=D+1}^{M} \sum_{n=D+1}^{M} \mathbf{e}_{m}^{+} \overline{\Delta G \mathbf{e}_{j} \mathbf{e}_{i}^{+} \Delta G} \mathbf{e}_{n} \mathrm{~d}^{+} \mathbf{e}_{m} \mathbf{e}_{n}^{+} \mathbf{d}
$$

which can be expressed compactly as

$$
\begin{aligned}
P_{1}(i, j) & =\mathrm{d}^{+} \mathrm{E}_{n} \mathrm{E}_{n}^{+} \overline{\Delta G e_{j} \mathrm{e}_{i}^{+} \Delta \mathrm{G}^{+}} \mathrm{E}_{n} \mathrm{E}_{n}^{+} \mathrm{d} \\
& =\mathrm{e}_{i}^{+} \overline{\Delta \mathrm{G}^{+} \mathbf{P}_{n} \mathrm{dd}^{+} \mathbf{P}_{n} \Delta \mathrm{G}} \mathrm{e}_{j},
\end{aligned}
$$

where $\mathbf{P}_{n}=\mathbf{E}_{n} \mathbf{E}_{n}^{+}$is the projection matrix onto the noise subspace. Since $\mathbf{d}$ is in the noise subspace, $\mathbf{P}_{n} \mathbf{d}=\mathbf{d}$. Therefore,

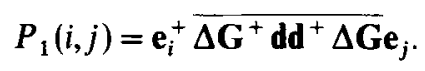

Thus, the $D \times D$ matrix $P_{1}$ becomes

$$
P_{1}=\mathrm{E}_{s}^{+} \overline{\Delta \mathrm{G}^{+} \mathrm{dd}^{+} \Delta \mathrm{G}} \mathbf{E}_{s}
$$

Since $\Delta g$ 's are uncorrelated from sensor to sensor, (51) can be simplified to

$$
P_{1}=\sigma_{\text {sum }}^{2} \mathbf{E}_{s}^{+}\left(\mathrm{dd}^{+}\right)_{\text {diag }} \mathbf{E}_{s},
$$

\footnotetext{
${ }^{1}$ Our method of obtaining the expression for the perturbation $\Delta d$ is much shorter and simpler than that of Rao \& Hari (1989b), and hence, it is included in the paper.
} 
where $\left(\mathrm{dd}^{+}\right)_{\text {diag }}$ denotes the diagonal matrix composed of the diagonal elements of $\mathrm{dd}^{+}$, and $\sigma_{\mathrm{sum}}^{2}=\sigma_{a}^{2}+\sigma_{p}^{2}$. Here $\sigma_{a}^{2}$ and $\sigma_{p}^{2}$ denote the variances of the sensor gain (amplitude) and phase errors, respectively. Thus, $\overline{\left|\Delta z_{i}\right|^{2}}$ can be expressed as

$$
\begin{aligned}
\overline{\left|\Delta z_{i}\right|^{2}} & =\left[M \sigma_{\text {sum }}^{2} / \prod_{l=1, l \neq i}^{M-1}\left|1-z_{l}^{-1} z_{i}\right|^{2}\right] \mathbf{V}^{+} E_{s} E_{s}^{+}\left(\mathrm{dd}^{+}\right)_{\text {diag }} \mathbf{E}_{s} \mathbf{E}_{s}^{+} \mathbf{V} \\
& =\left[M \sigma_{\text {sum }}^{2} / \prod_{l=1, l \neq i}^{M-1}\left|1-z_{l}^{-1} z_{i}\right|^{2}\right] \mathbf{V}^{+}\left(\mathrm{dd}^{+}\right)_{\text {diag }} \mathbf{V} .
\end{aligned}
$$

In arriving at the final form of (53), we have used the fact that $\mathbf{V}$ lies in the signal subspace and $E_{s} E_{s}^{+} V$ is its projection onto the signal subspace, and hence, remains as it is.

We now derive the expression for $\overline{\left(\Delta z_{i}\right)^{2}}$. From (42)

$$
\overline{\left(\Delta z_{i}\right)^{2}}=\left\{\exp \left(-j 2 \omega_{i}\right) M /\left[\prod_{l=1, l \neq i}^{M-1}\left(1-z_{l}^{-1} z_{i}\right)\right]^{2}\right\} \mathbf{V}^{+} \overline{\Delta \mathbf{d} \Delta \mathbf{d}^{T}} \mathbf{V}^{*}
$$

Substituting (A4) from appendix $A$ in (54), we get

$$
\overline{\left(\Delta z_{i}\right)^{2}}=\left\{\exp \left(-j 2 \omega_{i}\right) M /\left[\prod_{l=1, l \neq i}^{M-1}\left(1-z_{l}^{-1} z_{i}\right)\right]^{2}\right\} \mathbf{V}^{+} \mathbf{E}_{s} \overline{\Delta \mathbf{E}_{s}^{+} d d^{T} \Delta \mathbf{E}_{s}^{*}} \mathbf{E}_{s}^{*} \mathbf{V}^{*}
$$

Denoting $\overline{\Delta E_{s}^{+} d^{T} \Delta E_{s}^{*}}$ by $P_{2}$ and repeating the steps used above for evaluating $P_{1}$, we can show that

$$
\mathbf{P}_{2}=\sigma_{\text {diff }}^{2} \mathbf{E}_{s}^{+}\left(\mathbf{d d}^{T}\right)_{\text {diag }} \mathbf{E}_{s}^{*},
$$

where $\left(\mathrm{dd}^{T}\right)_{\text {diag }}$ denotes the diagonal matrix composed of the diagonal elements of $\mathrm{dd}^{T}$, and $\sigma_{\mathrm{diff}}^{2}=\sigma_{a}^{2}-\sigma_{p}^{2}$. Thus, $\overline{\left(\Delta z_{i}\right)^{2}}$ can be expressed as

$$
\begin{gathered}
\overline{\left(\Delta z_{i}\right)^{2}}=\left\{\exp \left(-j 2 \omega_{i}\right) M \sigma_{\text {diff }}^{2} /\left[\prod_{l=1, l \neq i}^{M-1}\left(1-z_{l}^{-1} z_{i}\right)\right]^{2}\right\} \times \\
\times \mathbf{V}^{+} \mathbf{E}_{\mathbf{s}} \mathbf{E}_{s}^{+}\left(\mathbf{d d}^{T}\right)_{\operatorname{diag}} \mathbf{E}_{s}^{*} \mathbf{E}_{s}^{T} \mathbf{V}^{*},
\end{gathered}
$$

which reduces to the following form since $\mathbf{E}_{\mathbf{s}} \mathbf{E}_{\mathbf{s}}^{+} \mathbf{V}=\mathbf{V}$ :

$$
\overline{\left(\Delta z_{i}\right)^{2}}=\left\{\exp \left(-j 2 \omega_{i}\right) M \sigma_{\text {diff }}^{2} /\left[\prod_{l=1, l \neq i}^{M-1}\left(1-z_{l}^{-1} z_{l}\right)\right]^{2}\right\} \mathbf{V}^{+}\left(\mathrm{dd}^{T}\right)_{\mathrm{dizg}} \mathbf{V}^{*}
$$

Combining (41) with the expressions for $\overline{\left|\Delta z_{i}\right|^{2}}$ and $\overline{\left(\Delta z_{i}\right)^{2}}$ derived above, and noting that $\Delta \omega=(2 \pi \mathrm{d} / \lambda) \cos (\theta) \Delta \theta$, we obtain the expression for MSE in the DOA estimates as

$$
\begin{aligned}
\overline{\left(\Delta \theta_{i}\right)^{2}}=\left[\frac{\lambda}{2 \pi d \cos \left(\theta_{i}\right)}\right]^{2} \frac{M}{2}\left[\frac{\sigma_{\text {sum }}^{2} \mathbf{V}^{+}\left(\mathbf{d d}^{+}\right)_{\text {diag }} \mathbf{V}}{\prod_{l=1, l \neq i}^{M-1}\left|1-z_{l}^{-1} z_{i}\right|^{2}}-\right. \\
\left.\quad-\operatorname{Re}\left\{\frac{\sigma_{\text {diff }}^{2} \mathbf{V}^{+}\left(\mathrm{dd}^{T}\right)_{\mathrm{diag}} \mathbf{V}^{*}}{\left[\prod_{l=1, l \neq i}^{M-1}\left(1-z_{l}^{-1} z_{i}\right)\right]^{2}}\right\}\right]
\end{aligned}
$$


The above expression holds for $D$ arbitrarily correlated (excluding the fully correlated case) signals. We now simplify (59) for the special case of a single source and obtain a simple and explicit expression for the MSE.

\subsection{One-source case}

For the case of a single source, $\mathbf{E}_{s}=(1 / \sqrt{M})\left[1, \exp \left(-j \omega_{1}\right), \ldots, \exp (-j)(M-1) \omega_{1}\right]^{T}$ and consequently

$$
\mathbf{g}=1 / \sqrt{M}, \quad \tilde{\mathbf{E}}_{\mathbf{s}}=\exp \left(-j \omega_{1}\right) \mathbf{V}^{\prime}
$$

Using (60) in (13), we get

$$
\tilde{d}=-\left\{\left(\exp \left[-j \omega_{1}\right] \sqrt{M}\right) /(M-1)\right\} \mathbf{V}^{\prime}
$$

Thus, the coefficient vector is given by

$$
\mathbf{d}=\left[\begin{array}{c}
1 \\
-\left[\exp \left(-j \omega_{1}\right) \sqrt{M} /(M-1)\right] \mathbf{V}^{\prime}
\end{array}\right] .
$$

Now consider the expression for $\mathbf{V}^{+}(\omega)$ d. Using the definition of $\mathbf{V}(\omega)$ and substituting $\exp (j \omega)=z$, we obtain

$$
\mathrm{V}^{+}(\omega) \mathrm{d}=\frac{1}{\sqrt{M}} \prod_{l=1}^{M-1}\left(1-z_{l}^{-1} z\right)
$$

Differentiating this expression with respect to $\omega$, it can be easily shown that at $\omega=\omega_{i}$,

$$
\mathrm{V}_{1}^{+} \mathrm{d}=-\frac{j}{\sqrt{M}} \prod_{l=1, l \neq i}^{M-1}\left(1-z_{l}^{-1} z_{i}\right)
$$

where $\mathrm{V}_{1}=(1 / \sqrt{M})\left[0,-j \exp \left(-j \omega_{i}\right), \ldots,-(M-1) j \exp \left(-j(M-1) \omega_{i}\right)\right]$. Using $(62)$, (64) and the definition of $\mathrm{V}\left(\mathrm{V}\left(\omega_{i}\right)\right)$, it can be shown (after some work) that at $\omega_{i}=\omega_{1}$, (59) reduces to

$$
\overline{\left(\Delta \theta_{1}\right)^{2}}=\left[\lambda / 2 \pi d \cos \left(\theta_{1}\right)\right]^{2} 4 \sigma_{p}^{2} /[M(M-1)] .
$$

Equation (65) shows that the MSE in the one-source case depends only on phase errors and is independent of the gain errors. A similar observation was made by Srinath \& Reddy (1991) for the MUSIC algorithm. The MSE expression for the case of MUSIC is given by

$$
\overline{\left(\Delta \theta_{1}\right)_{M U}^{2}}=\left[\lambda / 2 \pi d \cos \left(\theta_{1}\right)\right]^{2} 12 \sigma_{p}^{2} /[M(M-1)(M+1)] .
$$

From (65) and (66), we note that the root-Music ${ }^{2}$ is less sensitive to sensor phase perturbations than the minimum norm method when the number of sensors exceeds 2, i.e., $M \geqslant 3$.

\footnotetext{
${ }^{2}$ Though the expression derived by Srinath \& Reddy (1991) is not explicitly referred to the root-Music case, it is implicit because of the assumption of uniform linear array made in the development.
} 


\section{Simulation results}

In this section, we present some simulation results to demonstrate the validity of the analytical expressions derived in $\$ \$ 3$ and 4 . A uniform linear array with 8 isotropic elements with half-wavelength interelement spacing was used in the simulations. In the finite data case, 200 Monte Carlo runs were used when SNR $<6 \mathrm{~dB}$ while 50 runs were used when SNR $\geqslant 6 \mathrm{~dB}$. In the case of sensor gain and phase perturbations, we used 300 Monte Carlo runs throughout. The MSE was computed from the results obtained in different runs. The predicted value of the MSE was obtained by evaluating the corresponding expression [(59) or (65)].

\subsection{Finite data}

In the finite data case, simulations were done for both TLS-FBLP and minimum norm methods. In the one-source case the DOA of the source was kept at $10^{\circ}$. The SNR was varied from 0 to $20 \mathrm{~dB}$. The results are shown in figure 1 . Note from the plots that TLS-FBLP yields $3 \mathrm{~dB}$ lower MSE than the TLS-FLP method, as predicted in $\S 3$.

In the two-source case, the DOA were $18^{\circ}$ and $22^{\circ}$. As in the one-source case, the SNR was varied from 0 to $20 \mathrm{~dB}$. The MSE in the DOA estimate is shown for both DOA (see figure 2). The results, once again, confirm the theoretical prediction. The sudden increase in MSE indicates the threshold effect. Also, below the threshold SNR, the experimental performance deviates widely from that predicted, indicating that the first-order approximations used in the analysis are no longer adequate to explain the behaviour at these low SNR. We may point out here that the theoretical values in figures 1 and 2 for the minimum norm method were evaluated from the expressions given by Rao \& Hari (1989b) and $3 \mathrm{~dB}$ was added to these results to obtain the corresponding values for the TLS-FBLP case in accordance with the arguments in $\S 3$.

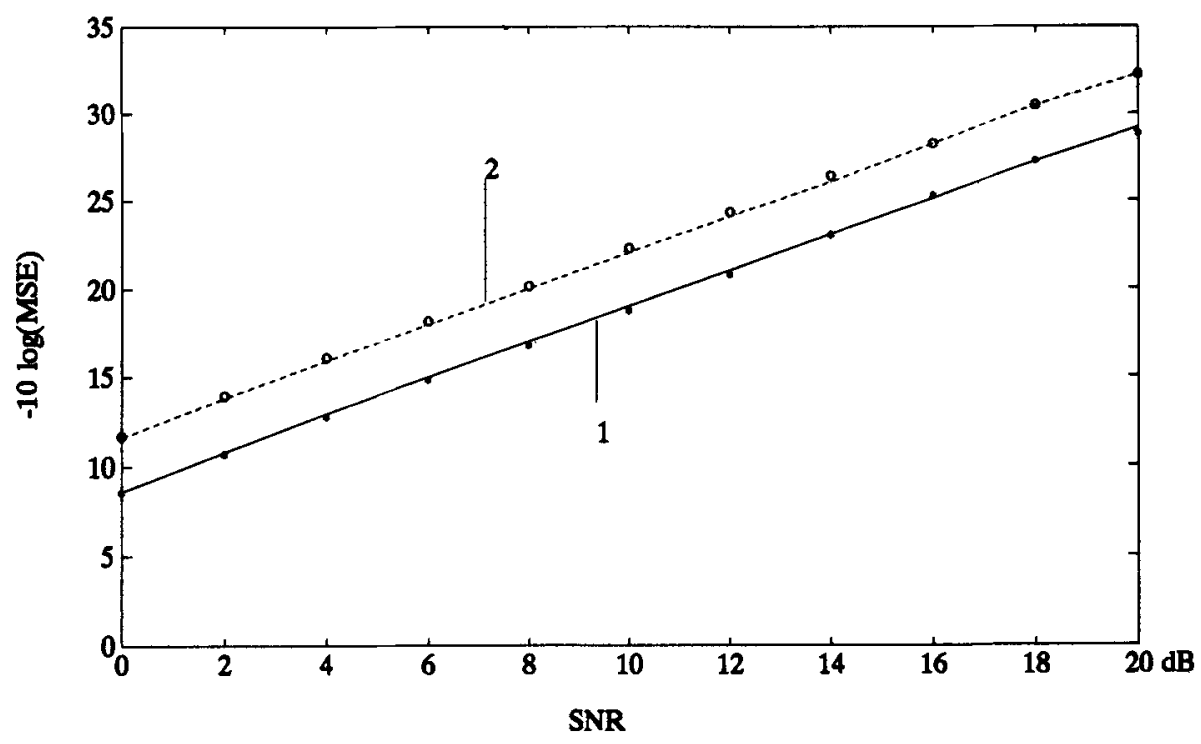

Figure 1. MSE performance in finite data for one-source case $\left(M=8, \theta=10^{\circ}\right.$, number of snapshots $(N)=100$. Plots 1 and 2 correspond to min. norm and TLS-FBLP methods, respectively, with ' $\star$ ' and ' $O$ ' denoting the corresponding simulated results). 

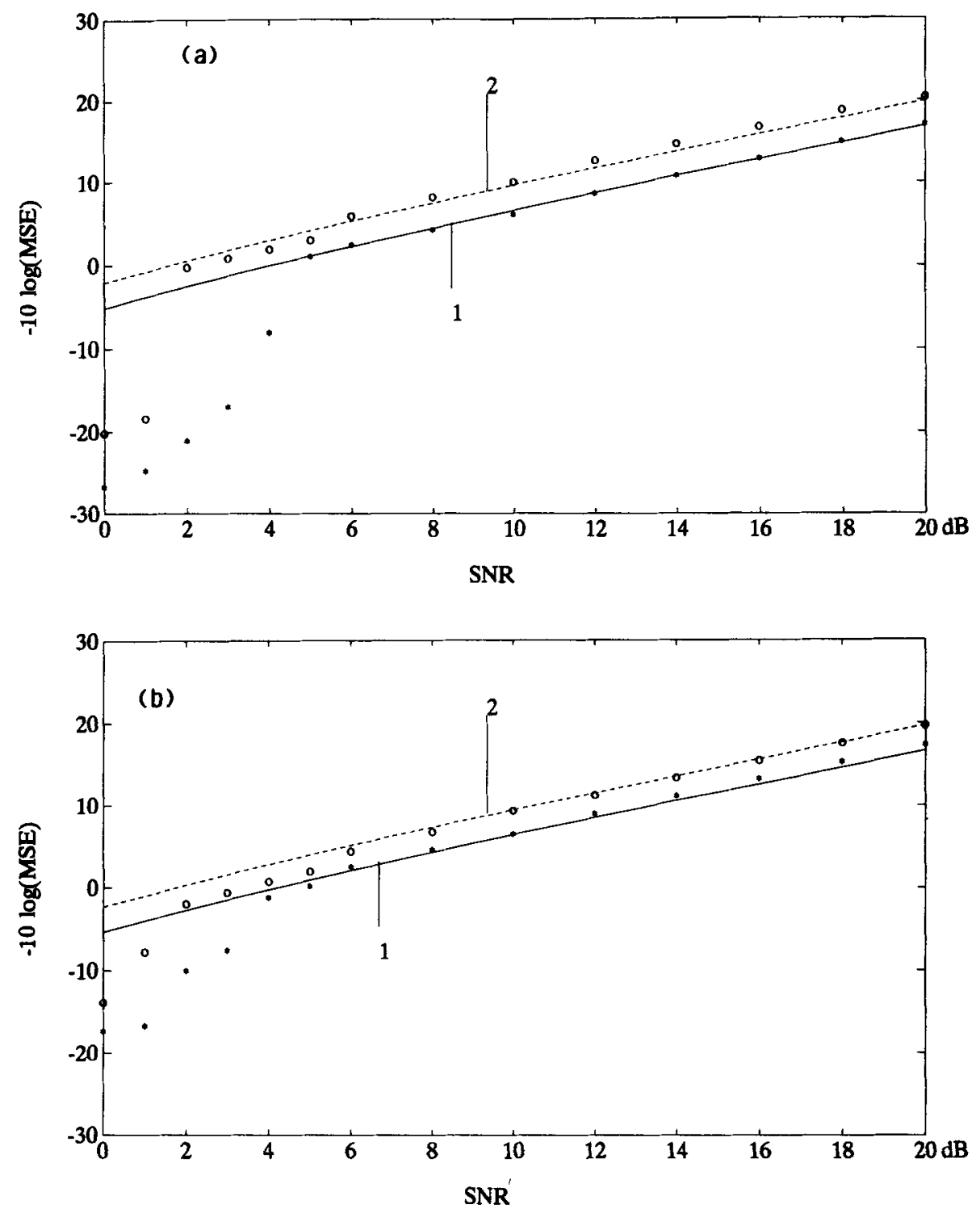

Figure 2. MSE performance in finite data for two-source case $\left(M=8, \theta_{1}=18^{\circ}, \theta_{2}=22^{\circ}\right.$, number of snapshots $(N)=100$. Plots 1 and 2 corrcspond to min. norm and TLS-FBLP methods, respectively, with ' $\star$ ' and ' $O$ ' denoting the corresponding simulated results); results for $\mathrm{DOA}=18^{\circ}(\mathrm{a})$ and for $\mathrm{DOA}=22^{\circ}(\mathrm{b})$.

\subsection{Sensor gain and phase errors}

In this case, simulations were made for the minimum norm method only (since the analysis developed was for the asymptotic case and the TLS formulations are restricted to finite data case only). The simulations were carried out for one-source and two-source cases. Errors considered in the sensors were either gain errors or phase errors, but not both. The array covariance matrix $\check{\mathbf{R}}$ was computed, assuming a single source with its DOA as $\theta$ or two sources with DOA as $\theta_{1}$ and $\theta_{2}$, as the case may be, with gain errors (or phase errors) as zero-mean uniform random variables in the 
Table 1. MSE (in degree $^{2}$ ) with phase errors only $\left(D=1, \mathrm{DOA}=10^{\circ}, M=8\right.$, SNR $=10 \mathrm{~dB}$ ).

\begin{tabular}{lcc}
\hline$\sigma_{p}^{2}$ & Simulated & Theoretical \\
\hline 0.25 & 0.0019 & 0.0019 \\
1.3333 & 0.0099 & 0.0099 \\
5.3333 & 0.0396 & 0.0398 \\
12 & 0.0890 & 0.0895 \\
21.333 & 0.1581 & 0.1592 \\
33.333 & 0.2467 & 0.2487 \\
48 & 0.3547 & 0.3582 \\
\hline
\end{tabular}

interval $[-\varepsilon, \varepsilon]$. The value of $\varepsilon$ was selected to give the required variance $\sigma_{a}^{2}$ (or $\sigma_{p}^{2}$ ). To be more explicit, consider the correlation matrix $\check{\mathbf{R}}$

$$
\check{\mathbf{R}}=\mathbf{G A S A}^{+} \mathbf{G}^{+}+\sigma^{2} \mathbf{I},
$$

where $\mathrm{G}$ is a diagonal matrix with elements $g_{i}=1+\Delta g_{i}$. In the case of gain errors only

$$
g_{i}=1+\Delta a_{i}
$$

and in the case of phase errors only

$$
g_{i}=\exp \left(j \Delta p_{i}\right)
$$

where $\Delta g_{i}$ and $\Delta p_{i}$ were modelled as zero-mean uniform random variables with variances $\sigma_{a}^{2}$ and $\sigma_{p}^{2}$. These errors were also assumed to be independent from sensor to sensor. From $\check{\mathbf{R}}$, the signal and noise subspaces were evaluated and the coefficient vector was computed. The DOA were estimated from the roots (which are closest to the unit circle) of the polynomial formed with this coefficient vector. This was repeated 300 times choosing the gain errors (or the phase errors) independently each time. From the 300 sets of the DOA estimates, the MSE was evaluated. Table 1 gives the

Table 2. MSE (in degree ${ }^{2}$ ) with amplitude errors only $\left(D=2, \mathrm{DOA}=10^{\circ}\right.$ and $20^{\circ}, M=8, \mathrm{SNR}=$ $10 \mathrm{~dB})$.

\begin{tabular}{lcc}
\hline$\sigma_{a}^{2}$ & Simulated & Theoretical \\
\hline $1.3333 \times 10^{-4}$ & 0.0039 & 0.0040 \\
& 0.0042 & 0.0044 \\
$5.333 \times 10^{-4}$ & 0.0155 & 0.0159 \\
& 0.0170 & 0.0174 \\
0.0012 & 0.0356 & 0.0357 \\
& 0.0391 & 0.0393 \\
0.0033 & 0.0984 & 0.0983 \\
& 0.1080 & 0.1079 \\
0.0133 & 0.4131 & 0.3961 \\
& 0.4519 & 0.4350 \\
0.03 & 1.0379 & 0.8934 \\
& 1.1270 & 0.9813 \\
\hline
\end{tabular}


Table 3. MSE (in degree $^{2}$ ) with phase errors only $\left(D=2, \mathrm{DOA}=10^{\circ}\right.$ and $20^{\circ}$, $M=8$, SNR $=10 \mathrm{~dB}$ ).

\begin{tabular}{lcc}
\hline$\sigma_{p}^{2}$ & Simulated & Theoretical \\
\hline 0.25 & 0.0017 & 0.0018 \\
& 0.0019 & 0.0020 \\
1.3333 & 0.0092 & 0.0096 \\
& 0.0102 & 0.0106 \\
5.3333 & 0.0370 & 0.0384 \\
& 0.0410 & 0.0422 \\
12 & 0.0838 & 0.0865 \\
& 0.0932 & 0.0950 \\
21.333 & 0.1503 & 0.1537 \\
& 0.1681 & 0.1689 \\
33.333 & 0.2492 & 0.2402 \\
& 0.2622 & 0.2638 \\
48 & 0.3649 & 0.3459 \\
& 0.3817 & 0.3799 \\
\hline
\end{tabular}

results for the one-source case while tables 2 and 3 give results for the two-source case. In tables 2 and 3 , the pair of entries for each value of $\sigma_{a}^{2}$ (or $\sigma_{P}^{2}$ ) corresponds to the MSE in the estimates of the two DOA.

In both the one- and two-source cases, we see that the agreement between the simulation results and the predicted values is good. Further, comparison with the corresponding results of MUSIC (see Srinath \& Reddy 1991) shows that the minimum norm method is more sensitive than MUSIC. This is what we predicted from the simplified analytical expression for the one-source case. We may also point out that a similar observation was made by Rao \& Hari (1989b) in the finite data case.

To see how the MSE varies with different array sizes for a given scenario, and with the source spacing for a given array size, we evaluated the theoretical expression for different values of $\sigma_{a}$ (or $\left.\sigma_{p}\right)$ (i) for two different array sizes $(M=8$ and 12), with DOA fixed at $10^{\circ}$ and $20^{\circ}$ and (ii) for two different sets of source locations (DOA $=10^{\circ}, 20^{\circ}$ and $10^{\circ}, 30^{\circ}$ ) with $M=8$, and plotted the results in figures 3 and 4, respectively. Note from figure 3 that the MSE decreases when the array size is increased. This is because when the array size increases, the source spacing in relation to the beamwidth of the array increases and, as a result, the sources tend to be treated as two separate single sources. That is, the array sees the two sources in two separate beams. Then, the MSE should tend to that of a single source for the corresponding value of $M$. In the case of gain errors, we know that the MSE is zero in the single source case. Hence, as the array size increases, the MSE should tend to zero. In the case of phase errors only, the expression for one-source case (cf. (65)) shows that the MSE decreases when $M$ is increased. This again explains why the MSE falls in the two-source case as the source spacing is increased keeping the array size fixed (see the plots in figure 4). Further, since the MSE in the single source case is zero for gain errors, the rate of fall in the MSE with increasing $M$ or source spacing will be faster in the case of gain errors than in the case of phase errors as shown by figures 3 and 4 . 

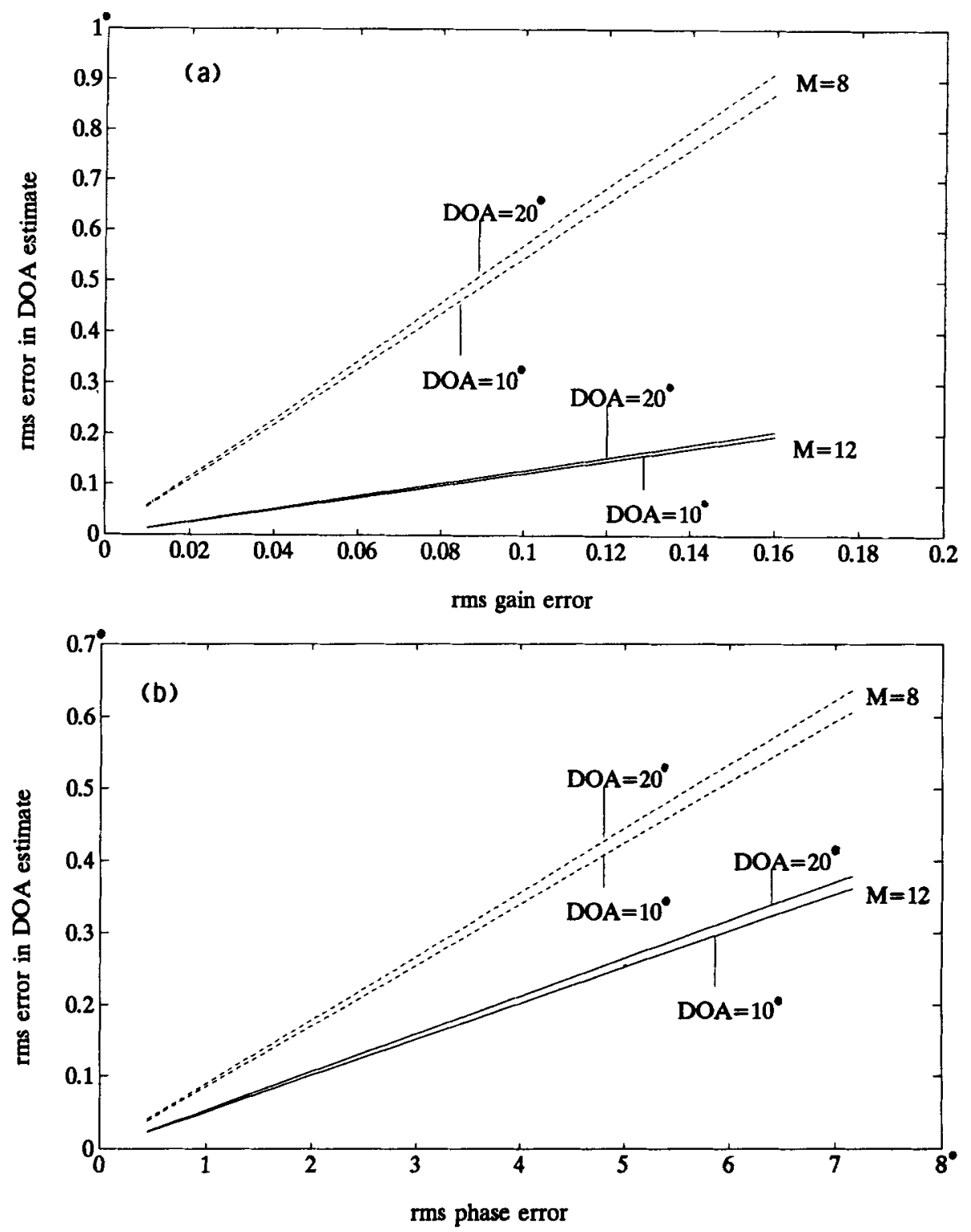

Figure 3. Asymptotic rms error performance of the min. norm method for two different array sizes, with gain errors only (a) and with phase errors only (b).

\section{Conclusions}

In this paper, we first considered the finite data performance and established two results: (i) The minimum norm method is equivalent to the TLS-FLP method. (ii) The TLS-FBLP method yields $3 \mathrm{~dB}$ lower MSE than the TLS-FLP method. We then took up the asymptotic performance of the minimum norm method in the presence of sensor gain and phase perturbations and derived expressions for the MSE in the DOA estimates. For the case of a single source, we obtained an explicit expression for the MSE which, 

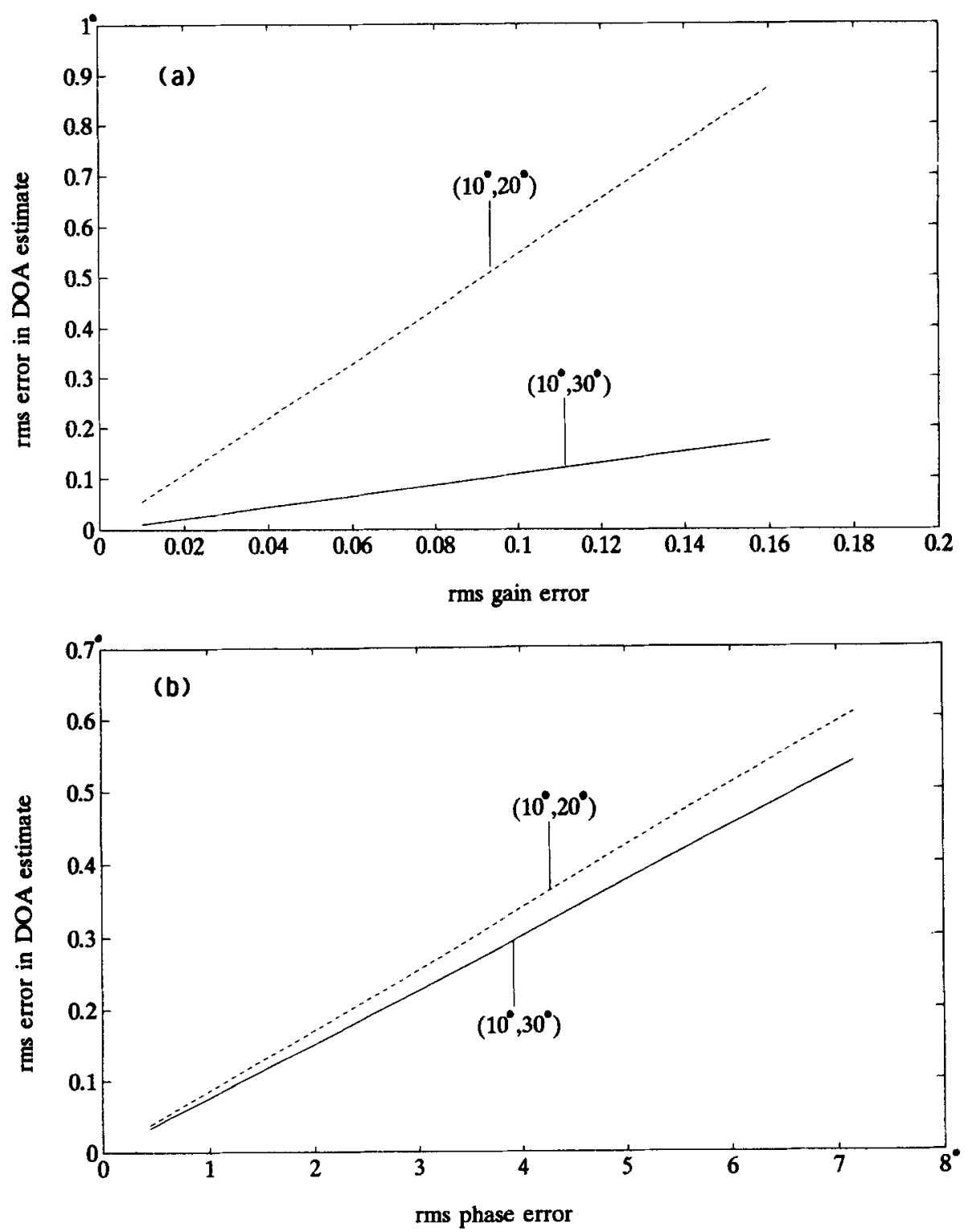

Figure 4. Asymptotic rms error performance of the min. norm method for two different source spacings (the plots are shown for $\mathrm{DOA}=10^{\circ}$ ), with gain errors only $(\mathrm{a})$ and with phase errors only (b).

when compared with the corresponding result for the MUSIC algorithm, shows that the minimum norm method is more sensitive than the Music. The same behaviour was confirmed for the two-source case by both the predicted values and the simulations.

This work is supported in part by the Electronics and Radar Development Establishment, Bangalore. 


\section{Appendix A}

In this appendix, we derive the expression for $\Delta d$, the perturbation in the coefficient vector $d$. Using the orthogonality of $d$ to the signal subspace, we have

$$
\left(\mathbf{E}_{s}+\Delta \mathbf{E}_{s}\right)^{+}(\mathbf{d}+\Delta d)=0 .
$$

Expanding (A1) and neglecting higher-order perturbations, we get

$$
\mathbf{E}_{s}^{+} \Delta \mathbf{d}+\Delta \mathbf{E}_{s}^{+} \mathbf{d} \approx 0,
$$

which can be rewritten as

$$
\mathbf{E}_{s}^{+} \Delta \mathbf{d} \approx-\Delta \mathbf{E}_{s}^{+} \mathbf{d}
$$

From (A3), we get

$$
\begin{aligned}
\Delta \mathbf{d} & \approx-\left(\mathbf{E}_{s}^{+}\right)^{\dagger} \Delta \mathbf{E}_{s}^{+} \mathbf{d} \\
& =-\mathbf{E}_{s} \Delta \mathbf{E}_{s}^{+} \mathbf{d},
\end{aligned}
$$

where the superscript ' $\uparrow$ ' denotes pseudoinverse.

\section{References}

Barabell A J 1983 Improving the resolution performance of eigenstructure-based direction finding algorithms. Proc. of ICASSP (Piscataway, NJ: IEEE Press)

Bienvenu G, Kopp L 1983 Optimality of high resolution array processing using the eigensystem approach. IEEE Trans. Acoust., Speech Signal Process. 31: 1235-1248

Golub G H, Van Loan C F 1980 An analysis of the total least squares troblem. SIAM J. Numer. Anal. 17: 883-893

Kaveh M, Barabell A J 1986 Statistical performance analysis of the MUSIC and minimum-norm algorithms in resolving plane waves in noise. IEEE Trans. Acoust., Speech Signal Process ASSP-34: 331-341

Kumaresan R, Tufts D W 1983 Estimating the angle of arrival of multiple plane waves. IEEE Trans. Aerosp. Electron. Syst. AES-19: 134-139

Paulraj A, Roy R, Kailath T 1985 Estimation of signal parameters via rotational invariance techniques ESPRIT. Proc. 19th Asilomar Conf. Circuits, Syst. Comput. (New York: IEEE Press)

Pisarenko V F 1973 The retrieval of harmonics from covariance functions. Geophys. J. R. Astronom. Soc. 33: $347-366$

Rahman M A, Yu K B 1987 Total least squares approach for frequency estimation using linear prediction. IEEE Trans. ASSP-35: 1440-1454

Rao B D, Hari K V S 1989a Performance analysis of root-MUSIC IEEE Trans. Acoust., Speech Signal Process ASSP-17: 1939-1949

Rao B D, Hari K V S 1989b Statistical performance analysis of the minimum-norm method. Proc. Inst. Electr. Eng. 136: 125-134

Reddy V U, Srinivas B 1989 Modified forward-backward linear prediction: Improvements and criterion for detection of the number of sinusoids. Special Issue on Advances in Statistical Signal Processing. Inst. Electron Telecommun. Eng. 35: 85-92

Schmidt R O 1986 Multiple source location and signal parameter estimation. IEEE Trans. Antennas Propag. 34: 276-280

Srinath H 1990 SVD-based superresolution alogithms: Finite data performance and analysis of MUSIC with sensor gain and phase perturbations, M E thesis, Dept. of Elect. Commun. Eng., Indian Institute of Science, Bangalore

Srinath H, Reddy V U 1991 Analysis of MUSIC with sensor gain and phase perturbation. Eur. Signal Process. 23: 245-256

Tufts D W, Kumaresan R 1982 Estimation of frequencies of multiple sinusoids: Making linear prediction perform like maximum likelihood. Proc. IEEE 70: 975-989 